\title{
Novel insights for SREBP-1 as a key transcription factor in regulating lipogenesis in a freshwater teleost, grass carp Ctenopharyngodon idella
}

\author{
Yi-Huan Xu ${ }^{1}$, Xiao-Ying Tan $^{1}$, Yi-Chuang Xu ${ }^{1}$, Tao Zhao ${ }^{1}$, Li-Han Zhang ${ }^{1}$ and Zhi Luo ${ }^{1,2 *}$ \\ ${ }^{1}$ Laboratory of Molecular Nutrition for Aquatic Economic Animals, Fishery College, Huazhong Agricultural University, Wuhan \\ 430070, People's Republic of China \\ ${ }^{2}$ Laboratory for Marine Fisheries Science and Food Production Processes, Qingdao National Laboratory for Marine Science and \\ Technology, Qingdao 266237, People's Republic of China
}

(Submitted 1 May 2019 - Final revision received 4 July 2019 - Accepted 31 July 2019)

\section{Abstract}

Disturbances in lipid metabolism are at the core of several health issues facing modern society, including fatty liver and obesity. The sterol regulatory element-binding protein 1 (SREBP-1) is one important transcription factor regulating lipid metabolism, but the relevant mechanism still remains unknown. The present study determined the transcriptional regulation of SREBP-1 and its target genes (including acetyl-COA carboxylase $\alpha(a c c \alpha)$, fatty acid synthase (fas) and stearoyl-CoA desaturase $1(s c d 1)$ ) in a freshwater teleost, grass carp Ctenopharyngodon idella. We cloned and characterised the $1988 \mathrm{bp}, 2043 \mathrm{bp}, 1632 \mathrm{bp}$ and $1889 \mathrm{bp}$ sequences of srebp-1, acc $\alpha, s c d 1$ and fas promoters, respectively. A cluster of putative binding sites of transcription factors, such as specific protein, yin yang 1 , nuclear factor $\mathrm{Y}$, sterol response elements (SRE) and enhancer box (E-box) element, were predicted on their promoter regions. Overexpression of nSREBP-1 reduced srebp-1 promoter activity, increased $s c d 1$ and fas promoter activity but did not influence $a c c \alpha$ promoter activity. The site-mutation and electrophoretic mobility shift assay analysis indicated that srebp-1, fas and $s c d$ promoters, but not acc $\alpha$ promoter, possessed SRE. In Ctenopharyngodon idella kidney (CIK) cells of grass carp, nSREBP-1 overexpression significantly reduced srebp-1 mRNA expression and up-regulated miR-29 mRNA expression. The $3^{\prime} \mathrm{UTR}$ of srebp-1 possessed the potential miR-29 binding site and miR-29 up-regulated the luciferase activity of srebp-1 $3^{\prime}$ UTR and srebp-1 mRNA expression, implying a self-activating loop of SREBP-1 and miR-29 in grass carp. Based on the above-mentioned results, we found two novel transcriptional mechanisms for SREBP-1 in grass carp: (1) the auto-regulation sited on the SREBP-1 promoter regions was suppressive and (2) there was a self-activating loop of SREBP-1 and miR-29.

\section{Key words: Ctenopharyngodon idella: Sterol regulatory element binding proteins: Promoter analysis: microRNA: Lipid} metabolism

Deregulated lipid metabolism is an established hallmark of many diseases, such as fatty liver and obesity. Lipid metabolism is tightly regulated by sterol regulatory element-binding protein 1 (SREBP-1), an important transcriptional factor that regulates the transcription of most genes involved in lipogenesis ${ }^{(1)}$. To date, two forms of mammalian SREBP-1 have been characterised, such as SREBP-1a and -1c. However, only a single form of the SREBP-1 gene has been characterised in fish ${ }^{(2,3)}$. The SREBPs are synthesised as precursor proteins bound to the endoplasmic reticulum membrane ${ }^{(4)}$. After stimulation, the SREBP precursor undergoes proteolytic cleavage in the Golgi to release the transcriptionally active $\mathrm{N}$-terminal domain (nSREBP). Once mature, the active SREBP1 translocates to the nucleus where it binds to sterol response elements (SRE) in the promoter regions of target genes to modulate their transcription ${ }^{(1,4,5)}$.

The target genes of SREBPs included SREBP itself, acetyl-CoA carboxylase $\alpha(\mathrm{ACC} \alpha)$, fatty acid synthase (FAS) and stearoylCoA desaturase (SCD1) ${ }^{(6,7)}$. ACC $\alpha$ catalyses the ATP-dependent carboxylation response of acetyl-CoA to form malonyl-CoA, and FAS catalyses the condensation of acetyl-CoA and malonyl-CoA to generate long-chain fatty acids ${ }^{(8)}$. SCD1 catalyses the synthesis of long-chain unsaturated fatty acids. At present, the promoter regions of these genes have been partially isolated and characterised in mammals ${ }^{(9-11)}$. Meantime, Griffin et al. pointed out that

Abbreviations: ACC $\alpha$, acetyl-CoA carboxylase $\alpha$; cDNA, complementary DNA; CIK, Ctenopharyngodon idella kidney; DMEM, Dulbecco's modified Eagle's medium; E-box, enhancer box; EMSA, electrophoretic mobility shift assay; FAS, fatty acid synthase; FBS, fetal bovine serum; miR, microRNA; NF-Y, nuclear factor Y; SCD1, stearoyl-CoA desaturase 1; SP, specific protein; SRE, sterol response elements; SREBP-1, sterol regulatory element-binding protein 1; UTR, untranslated region; WT, wild type; YY1, yin yang 1.

* Corresponding author: Z. Luo, emails luozhi99@mail.hzau.edu.cn; luozhi99@aliyun.com 
their regulation occurs mainly at the transcriptional level ${ }^{(12)}$. However, in fish, no reports investigated the mechanism of SREBP-1 regulating its target genes related to lipid metabolism.

On the other hand, the mechanisms involved in the activation of the SREBPs have been investigated in mammals ${ }^{(1,13)}$, but not in fish. MicroRNA (miR) are a class of small non-coding RNAs that regulate gene expression post-transcriptionally by binding to the complementary regions in the $3^{\prime}$ UTR of target mRNAs, resulting in mRNA degradation or attenuated translation ${ }^{(14)}$. Studies have shown that miR play a role in TAG homeostasis ${ }^{(15)}$. Recently, Ru et al. reported that SREBP-1 can activate miR-29 expression and in turn miR-29 inhibited srebp-1 expression, which unravelled a negative feedback loop mediated by miR-29 in SREBP-1 signalling ${ }^{(16)}$. However, in fish, no reports explore the molecular mechanism of SREBP regulation by miR-29.

Fish are by far the largest group of vertebrates in the world. Grass carp (Ctenopharyngodon idella) was an important herbivorous freshwater fish widely farmed all over the world. In some countries of European and Northern America, grass carp were widely used to control aquatic plants because of their aggressive feeding on vegetation ${ }^{(17)}$. Recently, the draft genome of the grass carp has been released, which is considered a convenient tool for identifying genomic structure of genes involved in lipid metabolism ${ }^{(18)}$. The present study was conducted to identify the promoter regions of $a c c \alpha$, fas, scd 1 and srebp-1 and investigate the functions of SRE on their promoter regions. To gain insight into the distinct roles of SREBP-1, overexpressing truncated, active nuclear forms of grass carp SREBP-1 were produced and characterised. The post-transcriptional regulation of srebp-1 by miR-29 was explored. Our study offers innovative insights into the regulatory mechanism of SREBP1 and provides direct evidence for SREBP-1 regulating itself and its downstream lipogenic genes in fish.

\section{Materials and methods}

\section{Animals, cells and media}

Juvenile grass carp were purchased from a commercial farm and used for DNA and RNA extraction. HepG2 and grass carp Ctenopharyngodon idella kidney (CIK) cell lines were obtained from our Cell Resource Center in Huazhong Agricultural University. HepG2 cells were cultured in Dulbecco's modified Eagle's medium (DMEM) containing $10 \%$ fetal bovine serum (FBS), penicillin $(100 \mathrm{U} / \mathrm{ml})$ and streptomycin $(0 \cdot 1 \mathrm{mg} / \mathrm{ml})$ in an incubator at $37^{\circ} \mathrm{C}$ with $5 \% \mathrm{CO}_{2}$. Grass carp CIK cells were cultured in Medium 199 (M199) containing 10\% FBS, penicillin $(100 \mathrm{U} / \mathrm{ml})$ and streptomycin $(0.1 \mathrm{mg} / \mathrm{ml})$ in an incubator at $28^{\circ} \mathrm{C}$ with $5 \% \mathrm{CO}_{2}$. All these culture media, $0.25 \%$ trypsinEDTA and FBS were purchased from Gibco (Thermo Fisher Scientific). Penicillin and streptomycin were purchased from Sigma-Aldrich. The present study was performed in accordance with the relevant institutional and national guidelines, and the manuscript must conform to the Animal Research: Reporting of In Vivo Experiments (ARRIVE) guidelines. The ethics committee of Huazhong Agricultural University checked and approved our present experimental protocols on animals and cells.

\section{Cloning and plasmids construction}

We identified the $5^{\prime}$ complementary DNA (cDNA) sequences and the transcription start sites of $s r e b p-1, a c c \alpha, s c d 1$ and fas of grass carp using RNA ligase-mediated rapid amplification of 5' cDNA ends (RLM-5'RACE) method. The promoter cloning was performed based on the published draft genome of grass carp $^{(18)}$, and the protocols followed these described in our recent studies $^{(19)}$. Genomic DNA was extracted from grass carp tail fins using a commercial kit (Tissue DNA Kit; Omega). The promoter sequences of $s r e b p-1, a c c \alpha, s c d 1$ and fas were amplified from extracted DNA using PCR and sub-cloned into pGl3-basic vector (Promega) using ClonExpress ${ }^{\mathrm{TM}}$ II One Step Cloning Kit (Vazyme), and the PCR were performed using TaKaRa PrimeSTAR ${ }^{\circledR}$ HS DNA Polymerase kit (TaKaRa). Based on the distance from their transcription start sites, we named the srebp1-1998 plasmid for -1998/+59 srebp-1 promoter, acc $\alpha$ 2043 plasmid for $-2043 /+49$ acc $\alpha$ promoter, scd1-1632 plasmid for $-1632 /+57 s c d 1$ promoter and fas-1889 plasmid for $-1889 /$ +111 fas promoter, respectively. Plasmids of srebp1-1493, srebp1-1098, srebp1-604, acc $\alpha-1538$, acc $\alpha-1069$, acc $\alpha-517$, scd1-1160, scd1-602, scd1-273, fas-1447, fas-1007 and fas-476, which contained unidirectional deletions of the promoter regions, were generated with the Erase-a-Base system (Promega) using templates of srebp1-1998, acc $\alpha-2043$, scd11632 and fas-1889 plasmid, respectively.

Total RNA was extracted from hepatic samples of grass carp using TRIzol reagent (Invitrogen) and reverse transcribed to cDNA as a template for constructing the nSREBP-1 expression plasmid and the $3^{\prime} \mathrm{UTR}$ of srebp-1 plasmid. The open reading frame sequence encoding nSREBP-1 was amplified from cDNA using PCR and sub-cloned into pcDNA3.1 (+) vector with the DYKDDDDK peptide (FLAG-tag) sequence inserted at the C-terminus of nsrebp-1 sequence using ClonExpress ${ }^{\mathrm{TM}}$ II One Step Cloning kit (Vazyme) and named as nSREBP-1 plasmid. For constructing $3^{\prime} \mathrm{UTR}$ of srebp-1 plasmid, the $3^{\prime} \mathrm{UTR}$ sequence of srebp-1 was amplified and sub-cloned into pmirGLO vector using ClonExpress $^{\mathrm{TM}}$ II One Step Cloning Kit (Vazyme) and named as pmirGLO-srebp1. All the primers were sequenced in a commercial company (Tsingke) and listed in online Supplementary Table S1.

\section{Sequence analysis}

Nucleotide sequences of srebp-1, acc $\alpha, s c d 1$ and fas promoters were compared with DNA sequences presented in the GenBank database (http://www.ncbi.nlm.nih.gov/genbank/) and the UCSC Genome Browser (http://genome.ucsc.edu/). For sequence analysis of the promoters of $a c c \alpha, f a s, s c d 1$ and srebp-1 from grass carp, putative transcription factor binding sites were predicted by online software MatInspector (http:// www.genomatix.de/). Besides, SRE with relative score over 0.9 based on JASPAR database (http://jaspar.genereg.net/) were also considered as potential binding sites.

\section{$m R N A$ and protein expression of grass carp nSREBP-1 in HepG2 cells}

For expression of nSREBP-1 plasmid group, HepG2 cells were counted and seeded at a density of $1 \times 10^{6}$ in a $60-\mathrm{mm}$ culture 
dish and then were cultured until 80-90\% confluence before transfecting with $8 \mu \mathrm{g}$ of nSREBP-1 plasmid using Lipofectamine 2000 (Invitrogen), according to the manufacture's instruction. For the control, the same amount of pcDNA3.1(+) was transfected into HepG2 cells. Then cells were harvested at 12-, 24- and 48-h incubation, respectively. Total RNAs from transfected HepG2 were extracted and reverse transcribed to cDNA with equal quantities of each total RNA $(1 \mu \mathrm{g})$ as templates for real-time quantitative PCR (Q-PCR). The resulting first-strand cDNA was diluted 1:10 with $\mathrm{ddH}_{2} \mathrm{O}$ before use. Q-PCR was performed using the SYBR Premix Ex TaqTM II kit (TaKaRa) in a quantitative thermal cycler (BIO-RAD). A set of six common housekeeping genes ( $\beta$-actin, 18s-rRNA, glyceraldehyde-3phosphate dehydrogenase (gapdh), elongation factor $1-\alpha$ (ef1 $\alpha$ ), hypoxanthine-guanine phosphoribosyl transferase ( $h p r t)$ and $\beta$-2-microglobulin $(b 2 m)$ ) were selected in order to test their transcription stability. Two most stable housekeeping genes were selected by geNorm software ${ }^{(20)}$. The relative expression levels were calculated with the delta-delta $\mathrm{Ct} \operatorname{method}^{(21)}$, when normalising to the geometric mean of the best combination of two housekeeping genes as suggested by geNorm. To confirm amplification specificity, the PCR products from each sample were examined by melting curve analysis. All experiments were performed in triplicates. These gene-specific primers for each gene are listed in online Supplementary Table S2.

Proteins for Western blot were extracted from transfected HepG2 cells using RIPA lysis and extraction buffer (Thermo Fisher Scientific), and the protocols followed these described in our recent studies ${ }^{(22)}$. Protein concentration was measured using the Pierce BCA protein assay kit (Thermo Fisher Scientific). About $30 \mu \mathrm{g}$ total protein per lane was loaded on SDS-PAGE and transferred to a polyvinylidene difluoride membrane. After blocked with $8 \%$ skimmed milk for $1 \mathrm{~h}$, the membranes were incubated with primary antibody at $4^{\circ} \mathrm{C}$ overnight. The primary antibodies used in the present study were rabbit polyclonal of anti-GAPDH (1:2000; Abcam) and anti-FLAG (1:1000; Proteintech). After washing for five times with Tris-buffered saline-Tween, the membrane was probed with horseradish peroxidase-conjugated anti-rabbit IgG (1:10000; Cell Signaling Technology) for $1 \mathrm{~h}$ at room temperature. The protein bands were visualised by Vilber Fusion FX6 Spectra imaging system (Vilber Lourmat) and quantified by Image-Pro Plus (Media Cybernetics).

\section{Luciferase assay of srebp-1, acc $\alpha$, scd 1 and fas promoters}

For promoter luciferase assays, HepG2 cells were counted and seeded at a density of $1 \times 10^{5}$ in twenty-four well plates, then cultured and transfected as mentioned in our recent studies ${ }^{(19,23)}$. Briefly, to study the nSREBP-1-induced changes in promoter activities, we co-transfected $300 \mathrm{ng}$ of nSREBP-1 plasmid or the same amount of pcDNA3.1(+) plasmid (300 ng, control) with $500 \mathrm{ng}$ of each of these luciferase reporter plasmids of srebp-1, $a c c \alpha, \quad s c d 1$ and fas promoters into HepG2 cells using Lipofectamine 2000 (Invitrogen) at 80-90\% confluence, respectively. For an internal control, 25 ng of Renilla luciferase vector the thymidine kinase promoter-Renilla luciferase reporter plasmid (pRL-TK) per well was included in all transfections to normalise transfection efficiency. At $6 \mathrm{~h}$ after the transfection, the cells were incubated with the fresh DMEM containing $10 \%$ FBS for $24 \mathrm{~h}$. Then the relative luciferase activity of srebp-1, acc $\alpha, s c d 1$ and fas promoters was measured using the Dual-luciferase Reporter Assay System (Promega), according to the manufacturer's instruction. The relative luciferase activity of these promoters was calculated using the ratio of Firefly luciferase activity:Renilla luciferase activity. All experiments were performed in triplicates.

\section{Site-mutation assay of sterol response elements sites on the srebp-1, acc $\alpha$, scd1 and fas promoters}

To identify the corresponding SRE on the grass carp srebp-1, $a c c \alpha, s c d 1$ and fas promoters, we performed site-directed mutagenesis of SRE on the plasmid of srebp-1, acc $\alpha, s c d 1$ and fas promoters, respectively, according to the manufacture's instruction of QuickChange II Site-Directed Mutagenesis kit (Vazyme). SREmutated plasmids of srebp-sre1, acc $\alpha$-sre1, scd1-sre1, scd1-sre2, fas-sre1, fas-sre2, fas-sre3, fas-sre 4 and fas-sre $3 \& 4$ were generated from the wild-type (WT) plasmids of srebp1-1998, acc $\alpha-$ 2043, scd1-1632 and fas-1889, respectively. Similar to the promoter luciferase assay performed previously, $300 \mathrm{ng}$ of nSREBP-1 plasmid or the same amount of pcDNA3.1(+) plasmid (300 ng, control) was co-transfected with $500 \mathrm{ng}$ of WT plasmid or the same amount of SRE-mutated plasmid into HepG2 cells using Lipofectamine 2000 (Invitrogen) at 80-90\% confluence. For an internal control, $25 \mathrm{ng}$ of Renilla luciferase vector (pRL-TK) per well was included in all transfections to normalise the transfection efficiency. At $6 \mathrm{~h}$ after the transfection, the cells were incubated with the same fresh DMEM containing $10 \%$ FBS for $24 \mathrm{~h}$. Then the cells were harvested and the relative luciferase activity was measured using the Dual-luciferase Reporter Assay System (Promega), according to the manufacturer's instruction. The relative luciferase activity of these promoters was calculated using the ratio of Firefly luciferase activity:Renilla luciferase activity. All experiments were performed in triplicates.

\section{Electrophoretic mobility shift assay}

An electrophoretic mobility shift assay (EMSA) was performed to confirm the functional SRE of the promoters. HepG2 cells were transfected with nSREBP-1 plasmid as described above. Nuclear proteins for EMSA were extracted from HepG2 cells, and concentrations were determined using Pierce BCA protein assay kit (Thermo Fisher Scientific). These extracts were stored at $80^{\circ} \mathrm{C}$ until analysed. Each oligonucleotide duplex of SRE was incubated with $5 \mu \mathrm{g}$ nuclear extracts at room temperature, according to the instruction of LightShift ${ }^{\mathrm{TM}}$ Chemiluminescent EMSA kit (Invitrogen), and each unlabelled probe was preincubated $10 \mathrm{~min}$ prior to the addition of biotin-labelled probe. The reaction was allowed to proceed for $20 \mathrm{~min}$ after the addition of biotin-labelled probe at room temperature and then were detected by electrophoresis on $6 \%$ native polyacrylamide gels. Competition analyses were performed using 200-fold excess of unlabelled oligonucleotide duplex with or without the SRE mutation. All the oligonucleotide sequences of EMSA were listed in online Supplementary Table S3. 
The prediction and luciferase assay of srebp-1 for miR-29

To validate the mRNA level of srebp-1 regulated by miR-29, we obtained the sequence of miR-29 of grass carp based on the recent publication ${ }^{(24)}$. The target sites of miR-29 on the $3^{\prime} \mathrm{UTR}$ of srebp-1 were predicted based on the principle of Targetscan ${ }^{(25)}$. The miR-29 targeted seed sequence was mutated on the WT pmirGLO-srebp1 plasmid using QuickChange II SiteDirected Mutagenesis kit (Vazyme), and the generated plasmid was named as pmirGLO-srebp1-miR29mut (Mut). Based on the site-mutation assay performed previously, 20 pmol of miR-29 mimics or the same amount of non-coding miR (20 pmol, negative control) was co-transfected with $500 \mathrm{ng}$ of the WT pmirGLOsrebp1 plasmid (WT) or 500 ng of pmirGLO-srebp1-miR29mut plasmid (Mut) into HepG2 cells using Lipofectamine 2000 (Invitrogen) at $90 \%$ confluence. At $6 \mathrm{~h}$ after the transfection, the cells were incubated, with the same fresh culture DMEM containing $10 \%$ FBS for $24 \mathrm{~h}$. Then the cells were harvested and the relative luciferase activity was measured using the Dual-luciferase Reporter Assay System (Promega), according to the manufacturer's instruction. The relative luciferase activity of these plasmids was calculated using the ratio of Firefly luciferase activity:Renilla luciferase activity. All experiments were performed in triplicates.

miR, $m R N A$ and protein expression induced by nSREBP-1 or miR-29 in Ctenopharyngodon idella kidney cells

Grass carp CIK cells were counted and seeded at a density of $1 \times 10^{6}$ in a $60-\mathrm{mm}$ culture dish. They were then cultured until $90 \%$ confluence was achieved before transfection using Lipofectamine 2000 (Invitrogen) with plasmids $(8 \mu \mathrm{g})$ or miR (100 pmol). Cells were harvested and washed with PBS. Then total RNA were extracted from the cells and reverse transcribed to cDNA as templates. Real-time quantification of miR was performed by stem-loop RT-PCR ${ }^{(26)}$, miR-29 stem-loop RT primers (5'-GTCGTATCCAGTGCAGGGTCCGAGGTATTCGCACTGGATACGACAACCGA-3') replaced oligo dT/random primers in Quantitect Reverse Transcription kit (TaKaRa). The resulting first-strand cDNA was diluted to 1:10 with $\mathrm{ddH}_{2} \mathrm{O}$ before use. The expression level of $\mathrm{miR}$ was determined by comparative delta-delta Ct method normalised with U6. These gene-specific primers are listed in online Supplementary Table S2. All experiments were performed in triplicates.

Proteins for Western blot were extracted from transfected cells using RIPA lysis and extraction buffer (Thermo Fisher Scientific). Protein concentration was measured using the Pierce BCA protein assay kit (Thermo Fisher Scientific). About $30 \mu \mathrm{g}$ total protein per lane was loaded on SDS-PAGE and transferred to a polyvinylidene difluoride membrane. After blocking with $8 \%$ skimmed milk for $1 \mathrm{~h}$, the membranes were incubated with primary antibody at $4^{\circ} \mathrm{C}$ overnight. The primary antibodies used in the present study were rabbit polyclonal of antiGAPDH (1:2000; Abcam) and rabbit polyclonal of anti-SREBP1 (1:1000; Abcam). After washing for five times with Tris-buffered saline-Tween, the membrane was probed with horseradish peroxidase-conjugated anti-rabbit IgG (1:10000; Cell Signaling Technology) for $1 \mathrm{~h}$ at room temperature. The protein bands were visualised by Vilber Fusion FX6 Spectra imaging system (Vilber Lourmat) and quantified by Image-Pro Plus (Media Cybernetics).

\section{Statistical analysis}

The data were analysed using SPSS 19.0 (SPSS Inc.). Results are presented as mean values with their standard errors for three independent experiments. Differences between two groups were assessed using the unpaired two-tailed Student's $t$ test unless otherwise noted. The differences were considered to be significant at $P<0.05$.

\section{Results}

Cloning and sequence analysis of the promoter regions of srebp-1, acc $\alpha$, scd 1 and fas

In the present study, we identified the transcription start sites of srebp-1 (GenBank accession no.: KJ162572), acco (GenBank accession no.: GU908475), scd1 (GenBank accession no.: AJ243835) and fas (GenBank accession no.: MK111644), and the first nucleotide of $\operatorname{srebp}-1, a c c \alpha$, fas and $s c d 1$ was designated as +1 . Then the 1988 bp, 2043 bp, 1632 bp and 1889 bp sequences of srebp-1, acc $\alpha, s c d 1$ and fas promoters were cloned and analysed, respectively. A cluster of putative binding sites of several transcription factors, such as specific protein (SP) family, yin yang 1 (YY1), nuclear factor $Y$ (NF-Y), SRE and enhancer box (E-box) element, were predicted on the promoters of srebp-1, $a c c \alpha, s c d 1$ and fas. On the region of srebp-1 promoter (online Supplementary Fig. S1), two SP binding sites (at $-60 \mathrm{bp} /-72 \mathrm{bp}$ and $-451 \mathrm{bp} /-467 \mathrm{bp}$ ), three YY1 binding sites (at $-515 \mathrm{bp} /-537$ bp, -1902 bp/-1924 bp and -1925 bp/-1947 bp), six NF-Y binding sites (at - 73 bp/-87 bp, -134 bp/-148 bp, - 485 bp/- 499 bp, -574 bp/ $-588 \mathrm{bp},-845 \mathrm{bp} /-859 \mathrm{bp}$ and $-970 /-984 \mathrm{bp}$ ), one SRE (at -597 bp/-611 bp) and one E-box element (at -508 bp/-524 bp) were predicted, respectively. On the region of $a c c \alpha$ promoter (online Supplementary Fig. S2), two SP binding sites (at -86 bp/-102 bp and $-116 \mathrm{bp} /-132 \mathrm{bp}$ ), one YY1 binding site (at $-436 \mathrm{bp} /$ $-458 \mathrm{bp}$ ), nine NF-Y binding sites (at $-62 \mathrm{bp} /-76 \mathrm{bp},-125 \mathrm{bp} /-$ 143 bp, -389 bp/-403 bp, -974 bp/-988 bp, -1186 bp/-1200 bp, $-1251 \mathrm{bp} /-1265 \mathrm{bp},-1360 \mathrm{bp} /-1374 \mathrm{bp},-1765 \mathrm{bp} /-1779 \mathrm{bp}$ and $-1960 \mathrm{bp} /-1974 \mathrm{bp}$ ), one SRE (at $-661 \mathrm{bp} /-675 \mathrm{bp}$ ) and two E-box elements (at -149 bp/-166 bp and -1232 bp/-1249 bp) were predicted, respectively. On the region of $s c d 1$ promoter (online Supplementary Fig. S3), there were two NF-Y binding sites (at $-950 \mathrm{bp} /-964 \mathrm{bp}$ and $-1040 \mathrm{bp} /-1054 \mathrm{bp}$ ), two SRE (at $-42 \mathrm{bp} /-56 \mathrm{bp}$ and $-1569 \mathrm{bp} /-1583 \mathrm{bp}$ ) and three E-box elements (at $-87 \mathrm{bp} /-103 \mathrm{bp},-714 \mathrm{bp} /-731 \mathrm{bp}$ and $-1477 \mathrm{bp} /-1494 \mathrm{bp}$ ). On the region of fas promoter (online Supplementary Fig. S4), we discovered one SP binding site (at $-1254 \mathrm{bp}-1270 \mathrm{bp}$ ), two YY1 binding sites (at -37 bp/-59 bp and - $1517 \mathrm{bp} /-1539 \mathrm{bp}$ ), seven NF-Y binding sites (at -90 bp/-104 bp, $-127 \mathrm{bp} /-141 \mathrm{bp},-785 \mathrm{bp} /$ $-799 \mathrm{bp},-1116 \mathrm{bp} /-1130 \mathrm{bp},-1147 \mathrm{bp} /-1161 \mathrm{bp}-1484 \mathrm{bp} /-1498 \mathrm{bp}$ and $-1685 \mathrm{bp} /-1699 \mathrm{bp}$ ), four SRE (at $-63 \mathrm{bp} /-72 \mathrm{bp},-133 \mathrm{bp} /$ $-142 \mathrm{bp},-1185 \mathrm{bp} /-1194 \mathrm{bp}$ and $-1235 \mathrm{bp} /-1249 \mathrm{bp})$ and four E-box elements (at $-55 \mathrm{bp} /-72 \mathrm{bp},-260 \mathrm{bp} /-276 \mathrm{bp},-270 \mathrm{bp} /$ $-286 \mathrm{bp}$ and $-1059 \mathrm{bp} /-1076 \mathrm{bp}$ ). 
(a)

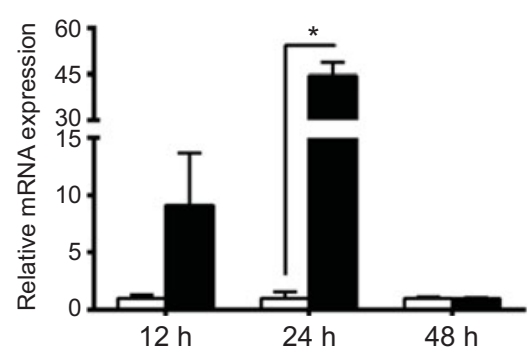

(b)

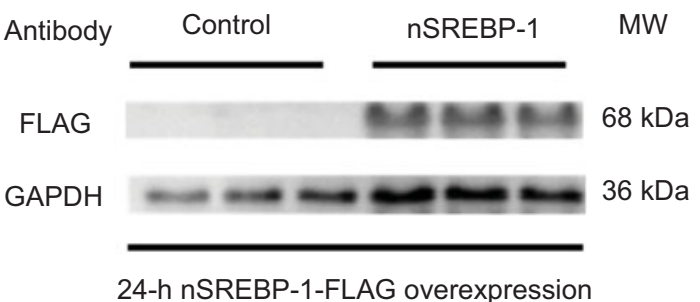

Fig. 1. Overexpression of the $\mathrm{N}$-terminal domain of sterol regulatory element-binding protein 1 (nSREBP-1) in HepG2 cells. (a) mRNA expression of $n S R E B P-1$ plasmid at 12, 24 and $48 \mathrm{~h}$ in HepG2 cells. ${ }^{*}$ Significant difference between nSREBP-1 overexpression $(\square)$ and control $(\square)$ groups $(P<0.05)$. $\beta$-Actin and glyceraldehyde-3phosphate dehydrogenase (gapdh) were chosen as the housekeeping genes $(M=0.345)$. (b) Western blot of FLAG antibody for nSREBP-1 in HepG2 cells at $24 \mathrm{~h}$. MW, molecular weight.

(a)

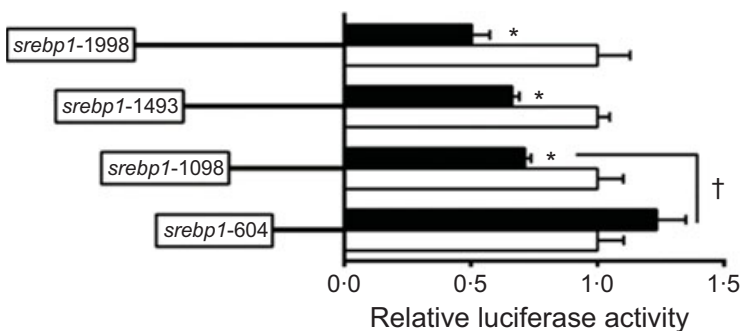

(c)

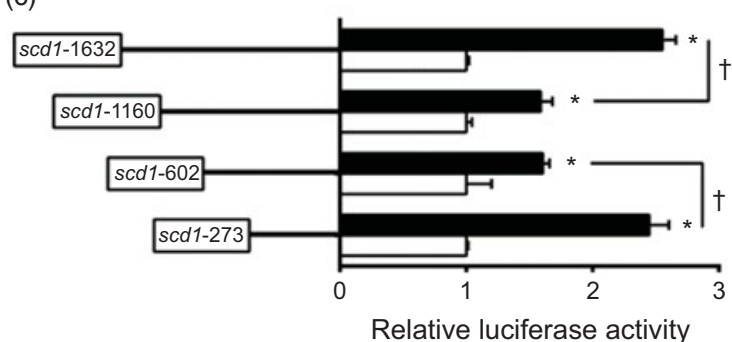

(b)

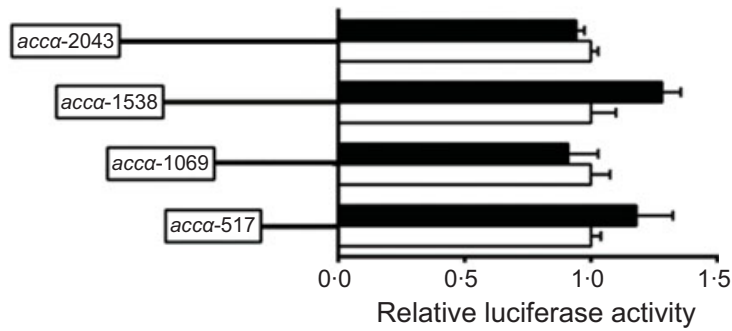

(d)

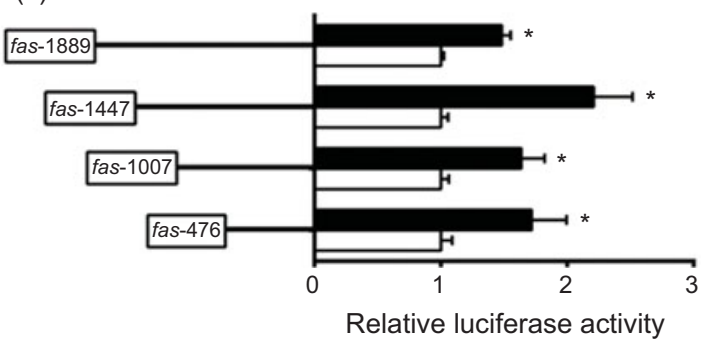

Fig. 2. 5'-Unindirectional deletion assays for promoter regions of sterol regulatory element-binding protein 1 (srebp-1), acetyl-CoA carboxylase $\alpha$ (acc $\alpha$ ), stearoyl-CoA desaturase 1 (scd1) and fatty acid synthase (fas) at $24 \mathrm{~h}$. (a) Assay for srebp-1 promoter region; (b) assay for acc $\alpha$ promoter region; (c) assay for scd1 promoter region; (d) assay for fas promoter region. Values are means $(n 3)$, with their standard errors represented by horizontal bars. * Significant difference in relative luciferase activities between the N-terminal domain of SREBP-1 (nSREBP-1) overexpression $(\square)$ and control $(\square)$ groups $(P<0.05)$. † Significant difference in nSREBP-1-induced changes in relative luciferase activity between the two promoter regions $(P<0.05)$. Relative luciferase activity was presented as the fold activated by nSREBP-1 compared with the control.

\section{Overexpression analysis of grass carp nSREBP-1 in HepG2 cells}

To reveal the mechanism of SREBP-1 regulating downstream target genes in grass carp, we tested the Dual-Luciferase Reporter system in cells derived from grass carp. However, compared with the canonical cells derived from mammals, the fish cells were not suitable for the Dual-Luciferase Reporter system, because the Renilla luciferase activities could not be detected. Thus, we chose HepG 2 cells for analysing the SREBP-1 function in lipogenic gene expression. Therefore, to investigate the nSREBP-1 plasmid for grass carp nSREBP-1 gene overexpressed in HepG2 cells, we determined the mRNA level of grass carp nSREBP-1 after transfection into HepG2 cells at 12-, 24- and $48 \mathrm{~h}$. Compared with the control, nSREBP-1 overexpression significantly up-regulated the mRNA and protein levels of
nSREBP-1 at $24 \mathrm{~h}$ (Fig. 1). Thus, to investigate the SREBP-1 function in gene expression, $24 \mathrm{~h}$ was used to determine the luciferase activities of $s r e b p-1, a c c \alpha$, fas and $s c d 1$ promoters.

\section{The $5^{\prime}$-deletion assay of the promoter regions of srebp-1, acc $\alpha, \operatorname{scd} 1$ and fas}

To investigate the activities of these promoters induced by nSREBP-1, we co-transfected nSREBP-1 plasmid along with the promoter constructs into HepG2 for $24 \mathrm{~h}$ and performed the $5^{\prime}$-deletion assay of srebp-1, acc $\alpha$, scd and fas promoters (Fig. 2). Compared with the control, overexpression of nSREBP-1 resulted in a reduction of srebp-1 promoter activity by $50 \%$, and the sequence deletion from -1998 bp to -1098 bp presented no significant influences on SREBP-1-induced promoter activity. However, further deleting the sequence 
(a)

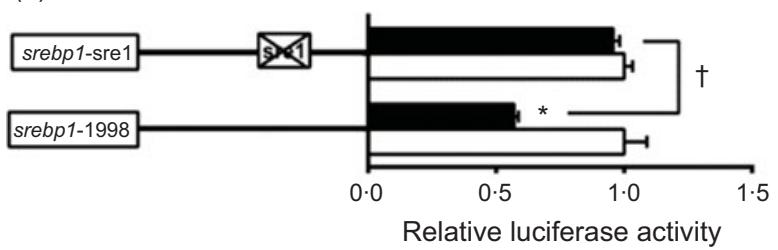

(c)

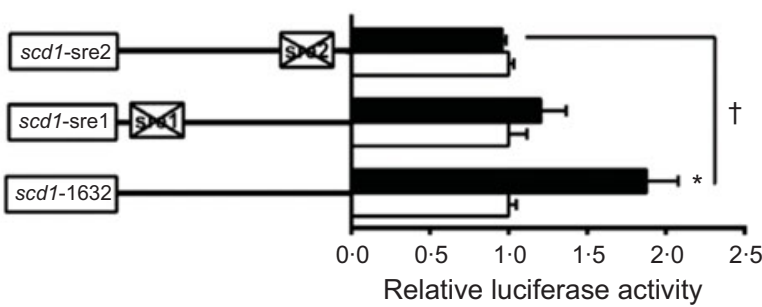

(b)

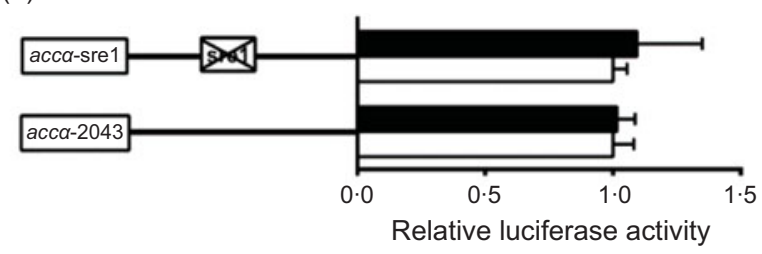

(d)

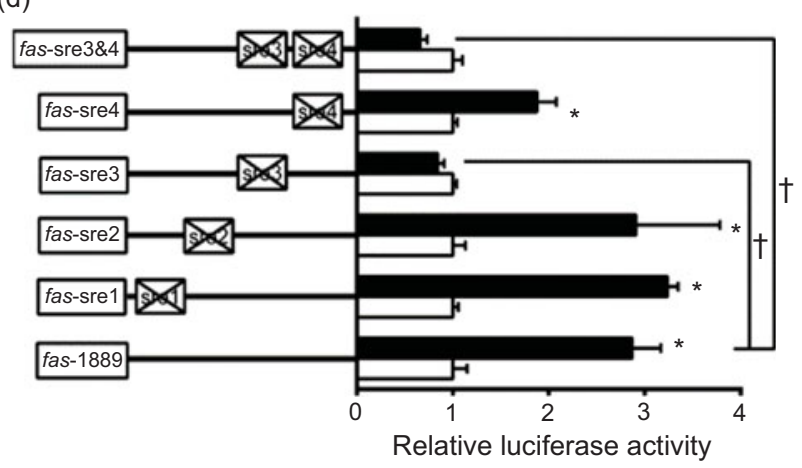

Fig. 3. Promoter activities of site mutagenesis on predicted sterol response elements at $24 \mathrm{~h}$. (a) Site mutagenesis on $-1998 /+58$ sterol regulatory element-binding protein 1 (srebp-1) promoter; (b) site mutagenesis on -2043/+49 acetyl-CoA carboxylase $\alpha$ (acc $\alpha$ ) promoter; (c) site mutagenesis on - 1632/+57 stearoyl-CoA desaturase 1 (scd1) promoter; (d) site mutagenesis on $-1889 /+111$ fatty acid synthase (fas) promoter. Values are means $(n 3)$, with their standard errors represented by horizontal bars. * Significant difference between the N-terminal domain of SREBP-1 (nSREBP-1) overexpression $(\square)$ and control $(\square)$ groups $(P<0.05)$. $†$ Significant difference in the nSREBP-1-induced changes of relative luciferase activity between two sites of mutagenesis $(P<0.05)$. The relative luciferase activity was presented as the fold activated by nSREBP-1 compared with the control.

between -1098 and -604 completely abolished the inhibitory effect by nSREBP-1, indicating that negative response element to nSREBP-1 existed on -1098/-604 region of srebp-1 promoter (Fig. 2(a)). Overexpressed nSREBP-1 showed no effect on the WT acc $\alpha$ promoter activity, and no significant differences were found in relative luciferase activity of $a c c \alpha$ promoter between different plasmid groups, indicating that the region of $a c c \alpha$ promoter was not influenced by nSREBP-1 (Fig. 2(b)). Overexpression of SREBP-1 markedly increased the $s c d 1$ promoter activity by $2 \cdot 6$-fold compared with the control. Deleting the sequence from $-1632 \mathrm{bp}$ to $-1160 \mathrm{bp}$ and from $-1160 \mathrm{bp}$ to -602 bp decreased the SREBP-1-induced $s c d 1$ promoter activity by $38 \%$, and further deleting the sequence between -602 bp to 273 bp recovered the SREBP-1-induced $s c d 1$ promoter activity, suggesting that $-1632 /-1160,-273 /-602$ and $-273 /+57$ region of $s c d 1$ promoter were influenced by nSREBP-1 (Fig. 2(c)). Overexpression of SREBP-1 significantly increased the fas promoter activity by $1 \cdot 5$-fold compared with the control, and further deleting the sequence from -1889 bp to -476 bp presented no significant effects on SREBP-1-induced fas promoter activity. These results indicated that there are positive responsive elements at $-476 /+111$ region of fas promoter to nSREBP-1 (Fig. 2(d)).

\section{Site-mutation analysis of sterol response elements on the} promoters of srebp-1, acc $\alpha$, fas and scd 1

To further elucidate whether the regions of srebp-1, acc $\alpha$, fas and $s c d 1$ promoters possessed SRE, we performed the site mutation at these regions of $s r e b p-1, a c c \alpha$, fas and $s c d 1$ promoters that potentially possessed SRE (Fig. 3). Overexpressed nSREBP-1 resulted in a reduction in srebp-1 promoter activity by $43 \%$ compared with the control, and its inhibitory effect was completely abolished when srebp1-sre1 (-597/-611) was mutated, suggesting that srebp1-sre1 site inhibited SREBP-1-induced srebp1 transcription (Fig. 3(a)). Consistent with $5^{\prime}$-deletion assays of the promoter region from -2043 to $+49 \mathrm{bp}$ of $a c c \alpha$, overexpressed nSREBP-1 showed no stimulatory effect on the WT acc $\alpha$ promoter activity, and mutation of acc1-sre1 (-661/-675) site did not affect SREBP-1-induced $a c c \alpha$ promoter activity, indicating that the $-2043 /+49$ region of $a c c \alpha$ did not possess any SRE (Fig. 3(b)). The promoter activity of WT scd 1 construct was enhanced by nSREBP-1 overexpression by 1.9-fold; mutation of scd1-sre2 (-42/-56) site completely abolished the stimulatory effect of SREBP-1, whereas mutation of scd1-sre1 (-1569/-1583) site showed no significant effect on SREBP-1-induced $s c d 1$ promoter activity, suggesting scd1-sre2 site up-regulated SREBP-1induced $s c d 1$ transcription (Fig. 3(c)). Overexpressed nSREBP1 markedly enhanced the WT fas promoter activity by $2 \cdot 9$-fold, but only the mutation plasmid of fas-sre 3 and fas-sre 3 and sre 4 , which were related to the mutation of fas-sre3 (-133/-142) site, caused a marked reduction in fas promoter activity and completely abolished the stimulatory effect of SREBP-1, indicating that fas-sre3 enhanced the SREBP-1-induced fas transcription (Fig. 3(d)).

\section{Electrophoretic mobility shift assay analysis of each SREBP-1 binding sequence}

Based on the results of the site-mutation assay discussed above, we further used EMSA to explore their ability to interact with SREBP-1. We made a probe using biotin to label the srebp1- 


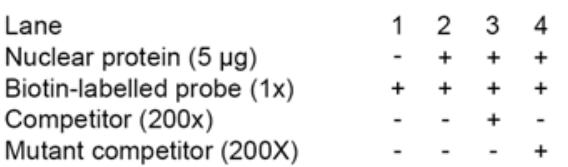

Mutant competitor (200X)
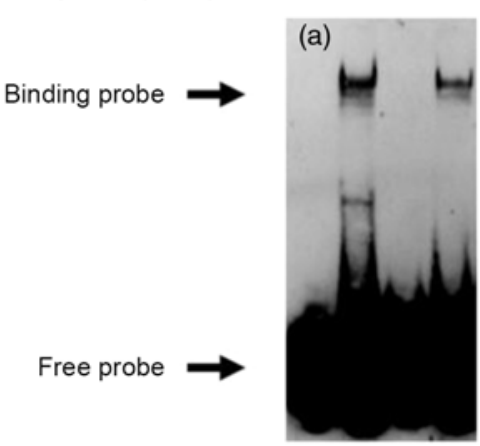

srebp1-sre1

(e)

Binding probe

sre1 sequence. The results indicated that the 200 -fold unlabelled srebp1-sre1 sequence competed the labelled probe for nSREBP1 and reduced the brightness of the labelled probe (lane 3, Fig. 4(a)). In contrast, the 200-fold unlabelled mutated srebp1-sre1 sequence did not compete for the labelled probe for nSREBP-1 (lane 4, Fig. 4(a)), indicating that srebp1-sre1 site could be bound by SREBP-1 (Fig. 4(a)). For acc $\alpha$-sre1 site of acc $\alpha$ promoter, the 200-fold unlabelled acc $\alpha$-sre1 sequence did not compete for the labelled probe, confirming that acc $\alpha$-sre 1 site could not interact with SREBP-1 (lane 3, Fig. 4(b)). Similarly, the unlabelled scd1-sre1 and scd1-sre2 sites of scd1 promoter did not compete for the labelled probe for nSREBP-1 (lane 3, Fig. 4(c) and (d)), indicating that scd1-sre2 site need a synergistic action of SREBP-1 assisted with other factors. In addition, EMSA analysis of four SRE on fas promoter indicated that the fas-sre1 (1235/-1249) site presented as a faint binding site of SREBP-1 (Fig. 4(e)); the unlabelled fas-sre2 (-1185/-1194) site and fas-sre4 site (-63/-72) did not compete for the labelled probe (lane 3, Fig. 4(f) and (h)). Only at the fas-sre3 (-133/-142) site (Fig. 4(g)), the 200fold unlabelled sequence competed for the labelled probe for SREBP-1 binding (lane 3, Fig. 4(g)) and the 200-fold mutated fas-sre3 did not compete for the labelled probe for nSREBP-1 binding (lane 4, Fig. 4(g)), indicating that fas-sre3 was a strong binding site for SREBP-1. Taken together, these results from
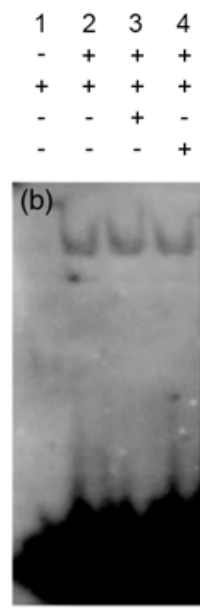

acca-sre 1

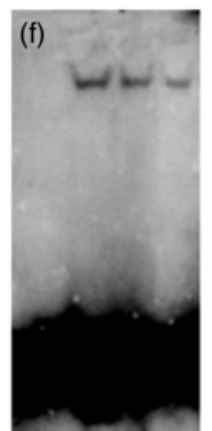

fas-sre2
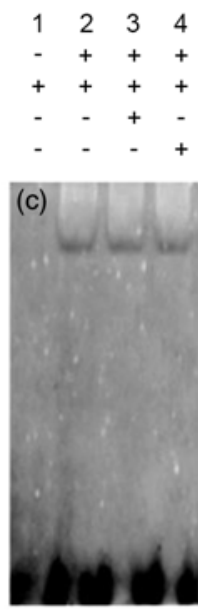

scd1-sre1

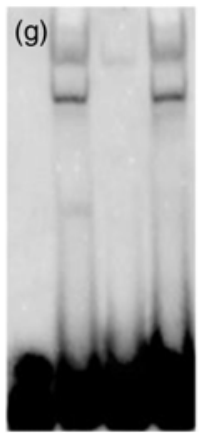

fas-sre3
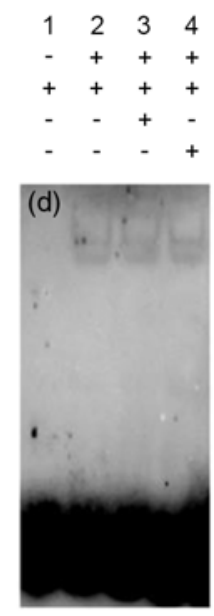

scd1-sre2

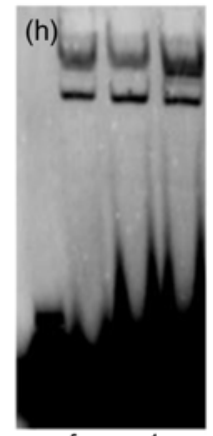

fas-sre4

Fig. 4. Electrophoretic mobility shift assay (EMSA) analysis of predicted sterol response elements (SRE). (a) -597/-611 binding site of sterol regulatory element-binding protein 1 (srebp-1) (srebp1-sre1); (b) -661/-675 binding site of acetyl-CoA carboxylase $\alpha$ (acc $\alpha$ ) (acc $\alpha$-sre1); (c) -1569/-1583 binding site of stearoyl-CoA desaturase 1 (scd1) (scd1-sre1); (d) -42/-56 binding site of scd1 (scd1-sre2); (e) -1235/-1249 binding site of fatty acid synthase (fas) (fas-sre1); (f) -1185/-1194 binding site of fas (fas-sre2); (g) -133/-142 binding site of fas (fas-sre3); (h) -63/-73 binding site of fas (fas-sre4).

luciferase activity, $5^{\prime}$-deletion and mutation analysis demonstrated that srebp-1, fas and $s c d 1$, but not $a c c \alpha$, were the target genes of SREBP-1.

\section{Analysis of nSREBP-1 overexpression in Ctenopharyngodon idella kidney cells}

The results above indicated that the promoter regions of genes involved in lipid metabolism (srebp-1, fas and scd 1) possessed SRE. These observations prompted us to investigate whether and how SREBP-1 regulated lipid metabolism in grass carp. We transfected the nSREBP-1 plasmid into CIK cells of grass carp and then determined the expression of its potential target genes and nSREBP-1. Compared with the control, the overexpression of nSREBP 1 significantly increased the mRNA expression of nSREBP-1, and significantly reduced srebp-1 mRNA expression by $30 \%$, and up-regulated mRNA expression of acc $\alpha$ and miR-29. mRNA expression of $f a s$ and $s c d 1$ tended to increase after nSREBP1 overexpression but the differences were not statistically significant between the two groups (Fig. 5(a)). Moreover, the protein level of nSREBP-1 tended to up-regulate during the transfection of nSREBP-1 plasmid into CIK cells, though the differences did not reach statistical significance (Fig. 5(b)). 
(a)

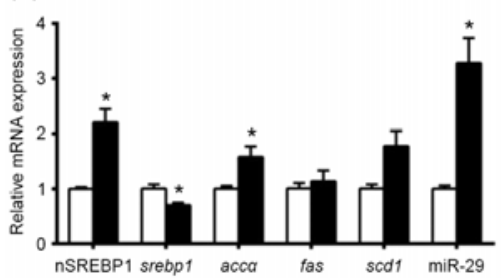

(b)

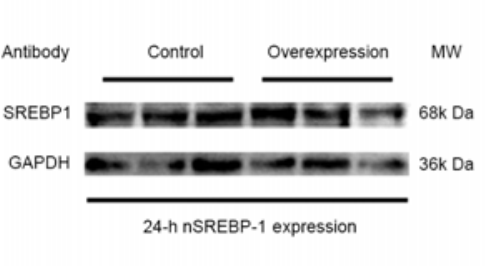

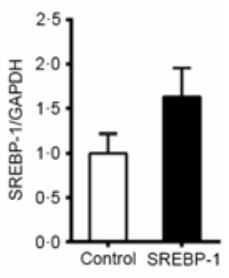

Fig. 5. Expression of sterol regulatory element-binding protein 1 (srebp-1), acetyl-CoA carboxylase $\alpha$ (acc $\alpha$ ), fatty acid synthase (fas) and stearoyl-CoA desaturase 1 (scd1) after N-terminal domain of SREBP-1 (nSREBP-1) overexpression in Ctenopharyngodon idella kidney (CIK) cells for $24 \mathrm{~h}$. (a) mRNA expression of srebp-1, acc $\alpha$, fas and scd1 and microRNA-29 (miR-29) in CIK cells for 24-h overexpression. $\beta$-Actin and elongation factor 1- $\alpha$ (ef1 $\alpha)$ were chosen as the housekeeping genes $(M=0.253)$. (b) Protein expression of nSREBP-1 in CIK cells for 24-h overexpression. Values are means $(n 3)$, with their standard errors represented by vertical bars. ${ }^{*}$ Significant differences between nSREBP-1 overexpression $(\square)$ and control $(\square)$ groups $(P<0.05)$. GAPDH, glyceraldehyde-3-phosphate dehydrogenase; MW, molecular weight.

(a)

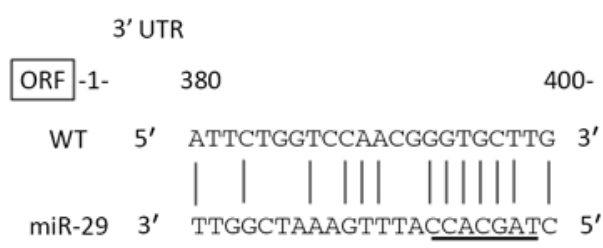

(b)

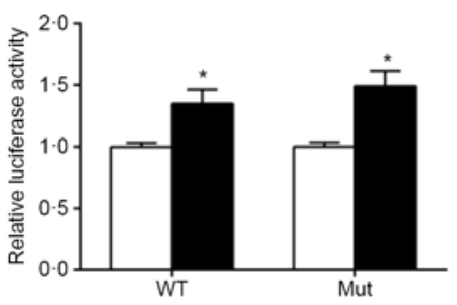

Fig. 6. Prediction and luciferase assay of $3^{\prime} U T R$ (untranslated region) of sterol regulatory element-binding protein 1 (SREBP-1) by microRNA-29 (miR-29). (a) Schematic representation of the miR-29 target sequence within the $3^{\prime} U T R$ of the srebp-1 gene. Vertical lines (I) indicate nucleotides that are reversely complementary to miR-29. The core seed of miR-29 is underlined. The numbers indicate the positions of the nucleotides in the srebp-13'UTR region. (b) Luciferase activities of the 3 'UTR of srebp-1 at $24 \mathrm{~h}$. Relative luciferase activity was presented as the fold activated by miR-29 mimics $(\square)$ compared with the negative control $(\square)$. Values are means $(n 3)$, with their standard errors represented by vertical bars. * Significant difference of luciferase activity between the miR-29 mimics and negative control groups $(P<0.05)$. WT, wild type.

(a)

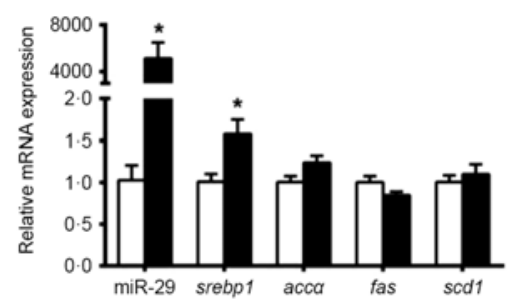

(b)

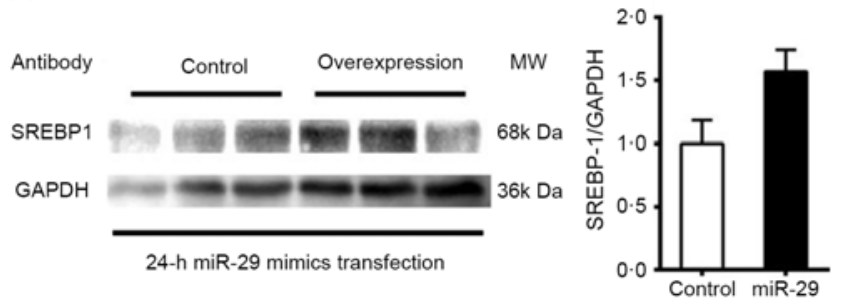

Fig. 7. Expression of sterol regulatory element-binding protein 1 (srebp-1), acetyl-CoA carboxylase $\alpha$ (acc $\alpha$ ), fatty acid synthase (fas) and stearoyl-CoA desaturase 1 (scd1) after microRNA-29 (miR-29) mimics transfection in Ctenopharyngodon idella kidney (CIK) cells for $24 \mathrm{~h}$. (a) mRNA expression of srebp-1 (nuclear part sequence of srebp-1), acc $\alpha$, fas and scd1 and miR-29 in CIK cells after miR-29 transfection at $24 \mathrm{~h} . \beta$-Actin and elongation factor $1-\alpha($ ef $1 \alpha)$ were chosen as the housekeeping genes $(M=0.242)$. (b) Protein expression of N-terminal domain of SREBP-1 (nSREBP-1) in ClK cells after miR-29 transfection at $24 \mathrm{~h}$. Values are means $(n 3)$, with their standard errors represented by vertical bars. * Significant difference between the nSREBP-1 overexpression $(\square)$ and control $(\square)$ groups $(P<0.05)$. GAPDH, glyceraldehyde-3-phosphate dehydrogenase; MW, molecular weight.

\section{Luciferase assay of 3'UTR of SREBP-1 by $\operatorname{miR}-29$}

The predicted miR-29 binding sites on the $3^{\prime} \mathrm{UTR}$ of srebp-1 are presented in Fig. 6(a). The luciferase reporter assay was used to verify whether miR-29 could influence the transcription of srebp-1. Compared with the negative control, co-transfection of miR-29 mimics with the pmirGLO-srebp1 significantly increased the luciferase activity of srebp-1 3'UTR by 1.3-fold, and its stimulatory effect was not abolished when the miR-29 targeted seed sequence of the srebp-1 3'UTR was mutated, indicating that miR-29 up-regulated the transcription of srebp-1 (Fig. 6(b)).

\section{Analysis of miR-29 transfection in Ctenopharyngodon idella kidney cells}

To support further the function of miR-29 in regulating the expression of SREBP-1 and its potential target genes, we transfected miR-29 mimics into the grass carp CIK cell lines. Compared with the negative control, miR-29 expression was significantly increased during the transfection of miR-29 mimics into CIK cells, and miR-29 mimics significantly increased the mRNA level of srebp-1 by 1.6-fold but showed no effect on the mRNA expression of $a c c \alpha$, fas and $s c d 1$ (Fig. 7(a)). Moreover, the protein level of nSREBP-1 tended to up-regulate during the 
transfection of miR-29 mimics into CIK cells, though the differences did not reach statistical significance (Fig. 7(b)).

\section{Discussion}

Several authors have overexpressed mature active SREBP-1 in fish, which activates the expression of target genes by translocating to the nucleus and binding to SRE within the promoters of target genes ${ }^{(6,27)}$. Here we prepared an $\mathrm{NH}_{2}$-terminal fragment of grass carp SREBP-1 (amino acid residues 1-494 of the protein, defined as nuclear SREBP-1, or nSREBP-1) and transfected the nSREBP-1 overexpression plasmid along with promoter reporter system to study the activation of potential target genes (srebp 1 , acc $\alpha$, fas and $s c d 1$ ).

In the present study, we cloned the $1988 \mathrm{bp}, 2043 \mathrm{bp}, 1632 \mathrm{bp}$ and 1889 bp sequences of $s r e b p-1, a c c \alpha, s c d 1$ and fas promoters, respectively. To our best knowledge, this is the first time to clone and characterise their promoter regions of these genes in fish. We predicted a cluster of putative binding sites of several transcription factors on the promoters of $s r e b p-1, a c c \alpha, s c d 1$ and fas of grass carp, such as SP, YY1, NF-Y, SREBP-1 itself and E-box element. Similar structures have been reported in mammals ${ }^{(13,28,29)}$. Sp1 has been shown to be a co-activating factor with SREBP-1a ${ }^{(30)}$. YY1 is a multifunctional $\mathrm{Zn}$-finger transcription factor that can act as a transcriptional repressor, activator or initiator element binding protein ${ }^{(10,31)}$. NF-Y was presented to be an essential co-activator of the sterol response ${ }^{(11,32)}$. The E-box is important for sterol regulation ${ }^{(33)}$. Accordingly, these transcription factor binding sites were important for its basal activation and also activation through other pathways.

The functional importance of SREBPs in controlling transcription of lipid metabolism-related genes is well established, but the mechanism remains unknown. The present study found that there were SRE on the promoters of $s r e b p-1, a c c \alpha, s c d 1$ and fas of grass carp. Multiple lines of evidence from $5^{\prime}$-deletion assay, site-mutation and EMSA analyses of each SRE on $a c c \alpha$, fas, $s c d 1$ and srebp-1 promoters further confirmed that those SRE actually presented as functional sites for SREBP-1 regulation and that srebp-1, fas and $s c d 1$, but not $a c c \alpha$, were the target genes of SREBP-1. Similarly, several studies suggested that the SRE- 1 and the E-box elements were existent in FAS promoter, which binds SREBP- $1^{(33)}$. Accordingly, SREBP-1 directly controls the expression of FAS $^{(11,34)}$. Li et al. ${ }^{(29)}$ reported that overexpression of SREBP-1 can increase FAS promoter activity and mRNA expression levels. SREBP-1 overexpression resulted in an increase in the mRNA levels of FAS and SCD ${ }^{(29,35)}$. Unexpectedly, based on the results from 5'-deletion assay, site-mutation and EMSA analyses of SRE on acc $\alpha$ promoters, the present study clearly indicated that $a c c \alpha$ was not the direct target gene of SREBP-1 though mammal's study found that SREBP-1 overexpression resulted in an increase in the mRNA levels of ACC ${ }^{(35)}$. Studies indicated that SREBPs required interaction with cofactors after binding to target DNAs to activate the downstream gene ${ }^{(5,33)}$. Similar results were also indicated by 5'-deletion assay, site-mutation and EMSA analyses of SRE on scd 1 promoter.

In mammals, studies suggested that SREBP-1 contained SRE in their enhancer/promoter regions, and the nuclear forms of
SREBPs can activate their own genes in an auto-regulatory loop ${ }^{(5,28,36)}$. However, in contrast with mammals, the present study clearly indicated that the activity of srebp-1 promoter was strongly inhibited by SREBP-1 itself. The reasons remained unknown now. Studies showed that SREBP activation of gene expression, in some SREBP target genes, can be negatively regulated by YY-1 Zn-finger transcription factor in manner of repressing SREBP activation by displacing NF-Y $\mathrm{Y}^{(37,38)}$. Moreover, YY1 could bind to SREBP-1 with a high affinity and interferes with the SREBP binding to the SRE on target genes ${ }^{(39)}$. Interestingly, we discovered that there are multiple potential YY1 binding sites on the promoter of srebp-1 gene of grass carp, but not on acc $\alpha$, fas and $s c d 1$ promoters. Thus, we concluded that it may be YY1 that causes the inhibition of srebp-1 expression during nSREBP-1 overexpression, indicating that a potential pathway might participate in SREBP-1-mediated lipid homeostasis.

In the present study, in grass carp CIK cells, the overexpression of nSREBP-1 significantly reduced srebp-1 mRNA expression and up-regulated mRNA expression of $a c c \alpha$. Thus, again our study confirmed that the SREBP-1 suppressed its own expression; meantime, it is the nuclear form of SREBP-1, not SREBP-1, that regulates its downstream target genes. Similarly, Kim et al. pointed out that SREBP1 translocates to the nucleus where it activates lipogenic genes by binding to the SRE of target genes $^{(7)}$. The present study also indicated that mRNA expression of $f a s$ and $s c d 1$, and the protein level of nSREBP-1 tended to upregulate during the transfection of nSREBP-1 plasmid into CIK cells but the differences did not reach statistical significance. We speculated that it may be because increased SREBP-1 protein expression was not enough to up-regulate mRNA expression of its target genes fas and $s c d 1$. It is well reported that some miR regulate the expression of target genes involved in lipid metabolism ${ }^{(15)}$. Recently, in mammals, $\mathrm{Ru}$ et al. reported that SREBP-1 transcriptionally activated specific SRE motifs on the promoter of miR-29, and then miR-29 inversely suppressed SREBP-1 expression by binding to their $3^{\prime} \mathrm{UTR}_{\text {region }}{ }^{(16)}$. In contrast, in the present study, we found that transfection of miR-29 mimics significantly increased the mRNA level of srebp-1 and miR-29 in CIK cells but showed no significant effects on the mRNA expression of $a c c \alpha$, fas and $s c d 1$. The up-regulation of srebp-1 mRNA level by miR-29 was not expected since miR are believed to bind through partial homologous sequence to a target gene at $3^{\prime} \mathrm{UTR}$ and cause translation repression. However, other studies suggest that miR can up-regulate translation of target genes ${ }^{(40)}$. On the other hand, we also noticed that miR-29 mimic transfection tended to up-regulate the protein expression of nSREBP1 and that nSREBP-1 overexpression up-regulated mRNA expression of miR-29, implying that a self-activating loop for SREBP-1 and miR-29 existed in grass carp. Further study is still needed to elucidate the details of the self-activating mechanism of SREBP-1 and miR-29 in grass carp.

\section{Conclusion}

In summary, we identified and characterised the promoter regions of $s r e b p-1, a c c \alpha$, fas and $s c d 1$ genes from grass carp. The present study demonstrated that $f a s$ and $s c d 1$ were the direct target genes of SREBP-1. Furthermore, we found two novel 
transcriptional mechanism for regulating SREBP-1 expression: (1) the auto-regulation sited on the SREBP-1 promoter regions was suppressive and (2) SREBP-1 overexpression up-regulated miR-29 expression, and SREBP-1 expression was up-regulated by miR-29 as well, implying a self-activating loop of SREBP-1 and miR-29 in grass carp. Our study shed us new sight into the regulation of lipid metabolism.

\section{Acknowledgements}

This work was supported by National Key R\&D Program of China (grant no. 2018YFD0900400), National Natural Science Foundation of China (grant no. 31422056) and Fundamental Research Funds for the Central Universities, China (grant no. 2662018PY089 to Z. L.).

Z. L. and Y.-H. X. designed the experiment; Y.-H. X. conducted the experiment with the help of X.-Y. T., Y.-C. X., T. Z. and L.-H. Z.; Y.-H. X., X.-Y. T. and Z. L. analysed the data; Y.-H. X. drafted the manuscript and Z. L. revised the manuscript. All the authors read and approved the manuscript.

The authors declare no conflicts of interest.

\section{Supplementary material}

For supplementary material/s referred to in this article, please visit https://doi.org/10.1017/S0007114519001934

\section{References}

1. Shao W \& Espenshade PJ (2012) Expanding roles for SREBP in metabolism. Cell Metab 16, 414-419.

2. Minghetti M, Leaver MJ \& Tocher DR (2011) Transcriptional control mechanisms of genes of lipid and fatty acid metabolism in the Atlantic salmon (Salmo salar L.) established cell line, SHK-1. BBA-Mol Cell Biol L 1811, 194-202.

3. Thomas JK, Wiseman S, Giesy JP, et al. (2013) Effects of chronic dietary selenomethionine exposure on repeat swimming performance, aerobic metabolism and methionine catabolism in adult zebrafish (Danio rerio). Aquat Toxicol 130, 112-122.

4. Brown MS \& Goldstein JL (1997) The SREBP pathway: regulation of cholesterol metabolism by proteolysis of a membranebound transcription factor. Cell $\mathbf{8 9}, 331-340$.

5. Amemiya-Kudo M, Shimano H, Hasty AH, et al. (2002) Transcriptional activities of nuclear SREBP-1a, -1c, and - 2 to different target promoters of lipogenic and cholesterogenic genes. J Lipid Res 43, 1220-1235.

6. Shimano H, Horton JD, Hammer RE, et al. (1996) Overproduction of cholesterol and fatty acids causes massive liver enlargement in transgenic mice expressing truncated SREBP-1a. J Clin Invest 98, 1575-1584.

7. Kim YM, Shin HT, Seo YH, et al. (2010) Sterol regulatory element-binding protein (SREBP) 1 mediated lipogenesis is involved in cell senescence. J Biol Chem 285, 29069-29077.

8. Leonard AE, Pereira SL, Sprecher H, et al. (2004) Elongation of long-chain fatty acids. Prog Lipid Res 43, 36-54.

9. Moustaid N, Beyer RS \& Sul HS (1994) Identification of an insulin response element in the fatty acid synthase promoter. J Biol Chem 269, 5629-5634.
10. Ericsson J, Jackson SM \& Edwards PA (1996) Synergistic binding of sterol regulatory element-binding protein and NF-Y to the farnesyl diphosphate synthase promoter is critical for sterol-regulated expression of the gene. J Biol Chem 271, 24359-24364.

11. Shimano H (2001) Sterol regulatory element-binding proteins (SREBPs): transcriptional regulators of lipid synthetic genes. Prog Lipid Res 40, 439-452.

12. Griffin MJ, Wong RH, Pandya N, et al. (2007) Direct interaction between USF and SREBP-1c mediates synergistic activation of the fatty-acid synthase promoter. J Biol Chem 282, 5453-5467.

13. Cagen LM, Xiong D, Wilcox HG, et al. (2005) Insulin activates the rat sterol-regulatory-element-binding protein 1c (SREBP-1c) promoter through the combinatorial actions of SREBP, LXR, Sp-1 and NF-Y cis-acting elements. Biochem J 385, 207-216.

14. Rottiers V, Najafi-Shoushtari SH, Kristo F, et al. (2011) MicroRNAs in metabolism and metabolic diseases. Cold Spring Harb Sym 76, 225-233.

15. Cui HY, Chen QL, Tan XY, et al. (2018) miR-205 mediated Cuinduced lipid accumulation in yellow catfish Pelteobagrus fulvidraco. Int J Mol Sci 19, 2980.

16. Ru P, Hu P, Geng F, et al. (2016) Feedback loop regulation of SCAP/SREBP-1 by miR-29 modulates EGFR signaling-driven glioblastoma growth. Cell Rep 16, 1527-1535.

17. Opuxzynski K \& Shireman KJV (1995) Herbivorous Fishes: Culture and Use for Weed Management. Bocan Raton, FL: CRC Press Inc.

18. Wang Y, Lu Y, Zhang Y, et al. (2015) The draft genome of the grass carp (Ctenopharyngodon idellus) provides insights into its evolution and vegetarian adaptation. Nat Genet 47, 625-631.

19. $\mathrm{Xu} \mathrm{YH,} \mathrm{Luo} \mathrm{Z,} \mathrm{Wu} \mathrm{K,} \mathrm{et} \mathrm{al.} \mathrm{(2017)} \mathrm{Structure} \mathrm{and} \mathrm{functional}$ analysis of promoters from two liver isoforms of CPT I in grass carp Ctenopharyngodon idella. Int J Mol Sci 18, 2405.

20. Vandesompele J, De-Preter K, Pattyn F, et al. (2002) Accurate normalization of real-time quantitative RT-PCR data by geometric averaging of multiple internal control genes. Genome Biol 3, R0034.

21. Pfaffl MW (2001) A new mathematical model for relative quantification in real-time RT-PCR. Nucleic Acids Res 29, e45.

22. Wei CC, Luo Z, Hogstrand C, et al. (2018) Zinc reduces hepatic lipid deposition and activates lipophagy via $\mathrm{Zn} 2+/ \mathrm{MTF}-1 /$ PPAR $\alpha$ and $\mathrm{Ca}^{2+} / \mathrm{CaMKK} \beta / \mathrm{AMPK}$ pathways. FASEB J 32, 6666-6680.

23. Wu K, Tan XY, Xu YH, et al. (2018) Functional analysis of promoters of genes in lipid metabolism and their transcriptional response to STAT3 under leptin signals. Genes $\mathbf{9}, 334$.

24. Gong W, Huang Y, Xie J, et al. (2017) Genome-wide identification and characterization of conserved and novel microRNAs in grass carp (Ctenopharyngodon idella) by deep sequencing. Comput Biol Chem 68, 92-100.

25. Lewis BP, Burge CB \& Bartel DP (2005) Conserved seed pairing, often flanked by adenosines, indicates that thousands of human genes are microRNA targets. Cell 120, 15-20.

26. Chen C, Ridzon DA, Broomer AJ, et al. (2005) Real-time quantification of microRNAs by stem-loop RT-PCR. Nucleic Acids Res 33, e179.

27. Chen G \& Zhang X (2010) New insights into s2p signaling cascades: regulation, variation, and conservation. Protein Sci 19, 2015-2030.

28. Amemiya-Kudo M, Shimano H, Yoshikawa T, et al. (2000) Promoter analysis of the mouse sterol regulatory element-binding protein-1c gene. J Biol Chem 275, 31078-31085.

29. Li J, Luo J, Xu H, et al. (2015) Fatty acid synthase promoter: characterization, and transcriptional regulation by sterol regulatory element binding protein-1 in goat mammary epithelial cells. Gene 561, 157-164. 
30. Sanchez HB, Yieh L \& Osborne TF (1995) Cooperation by sterol regulatory element-binding protein and $\mathrm{Sp} 1$ in sterol regulation of low-density lipoprotein receptor gene. J Biol Chem 270, 1161-1169.

31. Galvin KM \& Shi Y (1997) Multiple mechanisms of transcriptional repression by YY1. Mol Cell Biol 17, 3723-3732.

32. Xiong S, Chirala SS \& Wakil SJ (2000) Sterol regulation of human fatty acid synthase promoter I requires nuclear factor-Y-and Sp-1-binding sites. Proc Natl Acad Sci U S A 97, 3948-3953.

33. Bennett MK, Lopez JM, Sanchez HB, et al. (1995) Sterol regulation of fatty acid synthase promoter coordinate feedback regulation of two major lipid pathways. J Biol Chem 270, 25578-25583.

34. Magaña MM \& Osborne TF (1996) Two tandem binding sites for sterol regulatory element binding proteins are required for sterol regulation of fatty-acid synthase promoter. $J$ Biol Chem 271, 32689-32694.

35. Li N, Zhao F, Wei C, et al. (2014) Function of SREBP1 in the milk fat synthesis of dairy cow mammary epithelial cells. Int J Mol Sci 15, 16998-17013.
36. Sato R, Yang J, Wang X, et al. (1994) Assignment of the membrane attachment, DNA binding, and transcriptional activation domains of sterol regulatory element-binding protein-1 (SREBP-1). J Biol Chem 269, 17267-17273.

37. Bennett MK, Ngo TT, Athanikar JN, et al. (1999) Co-stimulation of promoter for low density lipoprotein receptor gene by sterol regulatory element-binding protein and Sp1 is specifically disrupted by the yin yang 1 protein. J Biol Chem 274, 13025-13032.

38. Ericsson J, Usheva A \& Edwards PA (1999) YY1 is a negative regulator of transcription of three sterol regulatory elementbinding protein-responsive genes. $J$ Biol Chem 274, 14508-14513.

39. Christenson LK, Osborne TF, McAllister JM, et al. (2001) Conditional response of the human steroidogenic acute regulatory protein gene promoter to sterol regulatory element binding protein-1a. Endocrinology 142, 28-36.

40. Vasudevan S, Tong Y \& Steitz JA (2007) Switching from repression to activation: microRNAs can up-regulate translation. Science 318, 1931-1934. 